\title{
BMJ Open Can promoting awareness of fetal movements and focusing interventions reduce fetal mortality? A stepped-wedge cluster randomised trial (AFFIRM)
}

\author{
Alexander E P Heazell, ${ }^{1,2}$ Christopher J Weir, ${ }^{3,4}$ Sarah J E Stock, ${ }^{5,6}$ \\ Catherine J Calderwood, ${ }^{7}$ Sarah Cunningham Burley, ${ }^{4} \mathrm{~J}$ Frederik Froen, ${ }^{8}$ \\ Michael Geary, ${ }^{9}$ Alyson Hunter, ${ }^{10}$ Fionnuala M McAuliffe, ${ }^{11}$ Edile Murdoch, ${ }^{12}$ \\ Aryelly Rodriguez, ${ }^{3,4}$ Mary Ross-Davie, ${ }^{13}$ Janet Scott, ${ }^{14}$ Sonia Whyte, ${ }^{5}$ \\ Jane E Norman ${ }^{5}$
}

To cite: Heazell AEP, Weir CJ, Stock SJE, et al. Can promoting awareness of fetal movements and focusing interventions reduce fetal mortality? A steppedwedge cluster randomised trial (AFFIRM). BMJ Open 2017;7:e014813. doi:10.1136/ bmjopen-2016-014813

- Prepublication history and additional material are available. To view these files please visit the journal online (http://dx.doi. org/10.1136/bmjopen-2016014813)

Received 21 October 2016 Revised 2 March 2017 Accepted 13 March 2017

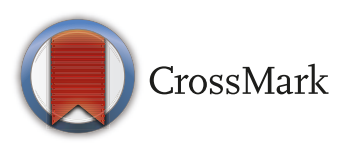

For numbered affiliations see end of article.

\section{Correspondence to} Alexander E P Heazell; alexander.heazell@manchester. ac.uk

\section{ABSTRACT}

Background In 2013, the stillbirth rate in the UK was 4.2 per 1000 live births, ranking 24th out of 49 high-income countries, with an annual rate of reduction of only $1.4 \%$ per year. The majority of stillbirths occur in normally formed infants, with (retrospective) evidence of placental insufficiency the most common clinical finding. Maternal perception of reduced fetal movements (RFM) is associated with placental insufficiency and increased risk of subsequent stillbirth. This study will test the hypothesis that the introduction of a package of care to increase women's awareness of the need for prompt reporting of RFM and standardised management to identify fetal compromise with timely delivery in confirmed cases, will reduce the rate of stillbirth. Following the introduction of a similar intervention in Norway the odds of stillbirth fell by $30 \%$, but the efficacy of this intervention (and possible adverse effects and implications for service delivery) has not been tested in a randomised trial.

Methods We describe a stepped-wedge cluster trial design, in which participating hospitals in the UK and Ireland will be randomised to the timing of introduction of the care package. Outcomes (including the primary outcome of stillbirth) will be derived from detailed routinely collected maternity data, allowing us to robustly test our hypothesis. The degree of implementation of the intervention will be assessed in each site. A nested qualitative study will examine the acceptability of the intervention to women and healthcare providers and identify process issues including barriers to implementation.

Ethics and dissemination Ethical approval was obtained from the Scotland A Research Ethics Committee (Ref 13/ SS/0001) and from Research and Development offices in participating maternity units. The study started in February 2014 and delivery of the intervention completed in December 2016. Results of the study will be submitted for publication in peer-reviewed journals and disseminated to local investigating sites to inform education and care of women presenting with RFM.

Trial registration number www.clinicaltrials.gov NCT01777022.

Version Protocol Version 4.2, 3 February 2017.
Strengths and limitations of this study

- This trial directly addresses the need for studies of the information given to women regarding fetal movements and the subsequent management of reduced fetal movements identified by Confidential Enquiries into Antepartum Stillbirths, Systematic Reviews and the Stillbirth Priority Setting Partnership.

- A stepped-wedge cluster trial design in combination with routinely collected maternity data allows the trial to be adequately powered to detect a difference in stillbirth as a primary outcome.

- The pragmatic nature of the study represents the potential impact of the introduction of such standardised care into clinical practice.

- The nested qualitative study will provide information regarding the acceptability of the intervention and identify barriers and facilitators to its adoption.

- The lack of information on resource use before and throughout the study period limits the ability to understand the consequences of the intervention on maternity unit workload.

\section{INTRODUCTION}

\section{Stillbirth}

Stillbirth, defined in the UK as a baby with no signs of life after 24 weeks of completed pregnancy, ${ }^{1}$ remains the major cause of perinatal mortality in high-income environments, with a recent series of papers in the Lancet on stillbirth issue calling for renewed action in this area. ${ }^{2}$ There is no single 'cause' of stillbirth, and a significant proportion of stillbirths remain unexplained, but fetal growth restriction (FGR), maternal hypertension and low socioeconomic status are among the identifiable risk factors. ${ }^{3}$

The concept that more can be done to reduce stillbirth in the UK and Ireland is supported by data showing a marked variation 
in rates between resource rich countries, when similar definitions of stillbirth are used. ${ }^{2}$ Notably, the UK has a higher rate than comparable resource rich countries such as Germany, Netherlands, New Zealand and Norway with rates in the UK some $50 \%$ greater than those of the Netherlands. Disappointingly, the annual rate of reduction in stillbirth from 2000 to 2014 in the UK was only $1.4 \%$ compared with $6.8 \%$ in the Netherlands and $2.8 \%$ in New Zealand. ${ }^{2}$ Rates of stillbirth in Scotland (3.7 per 1000 births in 2014) and Ireland (4.4 per 1000 live births in 2013) are similar to rates in England and Wales at 4.2 per 1000 live births (England and Wales, 2014). ${ }^{4}$ The reduction of avoidable harm for women and babies is viewed as a major priority for Government and its agencies throughout the UK and Ireland. Consequently, several initiatives have been developed by national governments in the UK and Ireland including the Scottish Government Stillbirth Working Group, NHS England Saving Babies' Lives Care Bundle and the Welsh Assembly 1000 Lives Plus strategy. These strategies have identified the need for better evidence to guide efforts to prevent stillbirths.

Using a robust priority setting strategy ${ }^{5}$ the Lancet Stillbirth's series steering committee identified issues around detection and management of reduced fetal movements (RFM) among the top 10 key research questions on prevention and management of stillbirth. ${ }^{6}$ This was confirmed in the UK-based Stillbirth Priority Setting partnership involving over 1700 parents and professionals which identified two relevant issues among the highest ranked research questions regarding stillbirth: (1) which investigations identify a fetus at risk of stillbirth after a mother believes she has experienced reduced fetal movements? and (2) would more accessible evidencebased information on signs and symptoms of stillbirth risk, designed to empower women to raise concerns with healthcare professionals, reduce the incidence of stillbirth? ${ }^{7}$ Thus, RFM has been identified as a highly relevant area of study by parents, professionals and researchers.

\section{RFMs, stillbirth and placental insufficiency}

There is a clear association between maternal perception of RFM and late stillbirth dating back over four decades. ${ }^{8}$ In a recent series of 2000 women, the adjusted OR (95\% CI) of late stillbirth in women with RFM (compared with controls) was 2.37 (1.29 to 4.35$).{ }^{9}$ One international study of 1714 women who experienced a stillbirth found that $30 \%$ had noted significant RFM prior to the diagnosis of stillbirth. ${ }^{10}$ Although the mechanisms have not been fully delineated, it is likely that RFM and stillbirth are linked by a common pathology, that of placental dysfunction. ${ }^{11}$ There is good evidence linking placental dysfunction and RFM. Compared with controls with an active fetus women who have fewer fetal movements on ultrasound scan immediately prior to caesarean section are more likely to have umbilical cord gas measurements indicative of acidaemia, hypoxaemia and hypercapnia. ${ }^{12}$ Women delivering within 1 week of an episode of RFM show differences in placental structure and function which are reminiscent of those seen in FGR and stillbirth. ${ }^{1314}$ Additionally, the odds of FGR (defined as being at less than the 10th centile for gestation adjusted birth weight) were greater in women with RFM compared with controls (adjusted OR 1.6, 95\% CI 1.1 to 2.2). ${ }^{15}$ Taken together these data are strong evidence that placental dysfunction is associated with RFM, and a causative pathway seems likely.

The evidence linking placental dysfunction and stillbirth is even stronger; a systematic review of placental pathology in stillbirths described abnormalities in up to $65 \%$ of cases. ${ }^{16}$ Among the 291 stillbirths in Scotland in 2010, 137 (47\%) had evidence of placental dysfunction. ${ }^{17}$ Given that the placenta was examined in only $80 \%$ of stillbirths, the true prevalence of placental dysfunction is likely to be higher. In addition, between $20 \%$ and $40 \%$ of stillborn babies are reported to have FGR, as defined by a birth weight less than the 10 th centile. ${ }^{18}$ Additionally, the Lancet report notes that "placental pathologies accounted for one in four deaths across all gestational ages, and were contributory or causal in more than half of cases. ${ }^{, 6}$ Given that stillbirth is strongly related to placental dysfunction, and RFM is a 'biomarker' of placental dysfunction then better management of women presenting with RFM focusing on the detection of placental dysfunction might reduce the risk of stillbirth.

\section{Formal fetal movement counting}

Although prenatal detection of FGR is improved by fetal movement counting, ${ }^{19}$ a systematic review, ${ }^{20}$ and a large and influential cluster randomised trial (which dominates the systematic review) showed that routine fetal movement counting using the count to 10 charts had no effect on perinatal mortality. ${ }^{21}$ Thus, the National Institute for Health and Social Care Excellence recommended that 'Routine formal fetal movement counting should not be offered. ${ }^{22}$ Importantly, the large cluster randomised trial tested a specific alarm limit for RFM, but did not recommend a specific management strategy for women who did present with RFM. There were two important observations from this study, first that in both groups the perinatal mortality rate was lower than contemporary or subsequent periods in the UK, and second that more women in the fetal movement counting arm came in with a live baby who subsequently died compared with the control arm (19 vs 11), suggesting that one reason the strategy failed to reduce perinatal mortality was inadequate investigation and management of those presenting with RFM. ${ }^{21}$

\section{Optimal strategy for determining RFM to prompt maternal presentation to the maternity service}

Maternal concern about RFM is a common reason to contact maternity services with between $6 \%$ and $15 \%$ of women presenting during the third trimester. ${ }^{23} 24$ Nevertheless, delays in reporting RFM to maternity care providers may increase the risk of adverse outcome. ${ }^{2526}$ The lack of good-quality information given to women 
about fetal movements has been highlighted as an example of suboptimal care in Confidential Enquiries into Antepartum Stillbirth. ${ }^{27}{ }^{28}$ Qualitative studies suggest that women frequently perceive RFM 2 days prior to the diagnosis of fetal death, and in some cases contractions were misinterpreted as fetal movements. ${ }^{29}$ Therefore, giving information to women regarding fetal movements and when they should be concerned about RFM is a key component of an intervention to reduce stillbirth.

However, giving clear information about RFM can be challenging as there is no uniform threshold of fetal movements below which perinatal morbidity increases, ${ }^{24}$ and no evidence that a specific threshold performs better than maternal perception of RFMs alone. ${ }^{8}$ Current guidelines from the Royal College of Obstetricians and Gynaecologists (RCOG) and Perinatal Society of Australia and New Zealand (PSANZ), ${ }^{30} 31$ informed by a large Norwegian study, ${ }^{32}$ suggest that it is maternal perception of decreased fetal movement which is important. Therefore, information for pregnant women in this study (shown in online supplementary file 1) described the importance of fetal movements, the need to get to know normal fetal activity, how fetal movements change in late pregnancy and who to contact if the mother perceives RFM. The educational package aimed to ensure that these messages were reinforced by staff behaviour at antenatal contacts.

\section{Optimal strategy for investigation and management of women presenting with RFM}

A recent systematic review found there are no proven strategies for the investigation and management of women presenting with RFM. ${ }^{33}$ Cardiotocography (CTG) is routinely used to ascertain fetal well-being, and it is the cornerstone of the RCOG guideline. ${ }^{31}$ However, data from Norway suggest that ultrasound assessment of fetal size is often the most helpful investigation, performing well on both an absolute basis, and compared with other interventions. ${ }^{34}$ In a series of over 3000 women with RFM, ultrasound (including measurement of fetal biometry and liquor volume) was found to be useful in detecting abnormalities in $11.6 \%$ of scans. In $71 \%$ of women in whom an abnormality was found, ultrasound was the only technique that detected an abnormality. Additionally, 85\% of abnormalities detected by ultrasound were important in informing the clinical management of the woman. ${ }^{34}$ These data are supported by a smaller UK study which found that abnormalities detected on CTG or ultrasound scan were most strongly associated with adverse outcome in women with RFM, with identification of abnormal estimated fetal growth centile on scan being the test most highly predictive of poor outcome. ${ }^{35}$ Perhaps this is not surprising, given the strong association between RFM and placental dysfunction and the central importance of ultrasound in the identification and management of small for gestational age babies. ${ }^{36}$ Given these data, it is concerning that a survey of clinicians in Scotland showed that fewer than $5 \%$ would routinely refer women with RFM for ultrasound examination (unpublished data from June 2012), and a survey of 223 UK midwives and obstetricians described that $17.9 \%$ of respondents would perform an ultrasound scan. ${ }^{37}$ These views of clinicians may reflect the variable quality of local guidelines, which are frequently not based on national recommendations, even those for which there is strong evidence. ${ }^{38}$ The variation in information given to women and subsequent management of RFM has been highlighted as sources of suboptimal care in two confidential enquiries into antepartum stillbirth. ${ }^{27}$ Therefore, we believe that current investigation of women presenting with RFM is inadequate, hence using the best available evidence, we have drafted what we consider to be a robust evaluation protocol for investigation of women with RFM.

\section{Potential efficacy of a package of intervention for RFM}

Supportive data for the package of interventions used in this study (information for women and standardised management protocol) come from a large observational 'clinical quality improvement study' in Norway which found a significant fall in rates of stillbirth (from 3.0/1000 to $2.0 / 1000$ (OR $0.67,95 \%$ CI 0.48 to 0.93 )) after the introduction of an intervention package consisting of written information for women about awareness of RFM combined with consensus guidelines for health professionals about their management. ${ }^{32}$ Although this study was not randomised, and therefore constitutes only level II-3 evidence, it has informed recommendations from the RCOG and PSANZ that 'women should be advised to be aware of their baby's individual pattern of movements and that if they are concerned about a reduction in or cessation of fetal movements... they should contact their maternity unit. ${ }^{3031}$ Following initial publication of the Norwegian study, a reanalysis was required as discrepancies between stillbirth rates in the study and the Medical Birth Registry of Norway were identified. This reanalysis found the reduction in stillbirth rates was of borderline statistical significance (OR $0.72,95 \%$ CI 0.50 to 1.03 ). The authors concluded that further studies were needed to determine whether this approach was associated with a reduction in stillbirth. ${ }^{39}$

Importantly, in the Norwegian study, there was no increase in the proportion of women who presented with RFM when rates were compared before and after the intervention. ${ }^{32}$ However, women with RFM presented significantly earlier to hospital than they had hitherto, potentially allowing time for intervention to reduce perinatal mortality. These data suggest that a package of interventions encouraging women with RFM to present early to hospital, combined with a structured approach to their management might reduce rates of stillbirth without contributing to a large increase in admissions antenatally.

\section{Potential harms of a package of care around increased awareness and optimised management of RFM}

Any clinical intervention which aims to improve outcomes also has the ability to do harm. Thus, it is essential that the intervention proposed is rigorously evaluated using 
the gold standard technique of a randomised trial, rather than being introduced as a service development. When the study began, there was a small window of opportunity to do this, as the enthusiasm to improve current management of RFM is such that routine introduction of the package of care is unlikely to be delayed much further than the current scheduled end date of this study. Possible harms of a package of care consisting of a management plan for identification and delivery of the 'at risk' fetus, together with strategies for increasing pregnant women's awareness of the need to report early include increased maternal anxiety and increased intervention (including hospital admission, induction of labour and caesarean section) which itself is associated with pregnancy-related complications. The available evidence is reassuring on some of these issues. A systematic review of 23 publications from 16 studies found three studies involving 2030 women addressing maternal concern and an additional three studies involving 1468 women investigating maternal-fetal attachment. These demonstrated no evidence of increased maternal anxiety and results regarding maternal-fetal attachment were discordant. ${ }^{40}$ In the Norwegian service development study, the package of care increased rates of follow-up of women, but there was no increase in admissions overall, admissions for induction or admissions for emergency caesarean section ${ }^{32}$-again, while reassuring these outcomes require formal evaluation in a randomised and relevant setting to the UK and Ireland. The final possible harm of the package is around increased resource use, and the opportunity cost of focusing on RFM rather than other potential methods to prevent stillbirth.

\section{RATIONALE}

The aim of this study is to test the hypothesis that a package of interventions consisting of strategies for increasing pregnant women's awareness of the need to report early when they perceive a reduction in fetal movements, followed with a management plan for identification and delivery of the 'at risk' fetus in such women, will reduce rates of stillbirth.

\section{STUDY OBJECTIVES}

\section{Primary objective}

The primary objective is to answer the research question 'Does the introduction of a protocol for detection and management of decreased fetal movements reduce rates of stillbirth?'

The secondary objectives are to answer the following research questions:

- What is the effect of the intervention on rates of caesarean section and induction of labour?

- What is the effect of the intervention on rates of admission to the neonatal intensive care unit?
- What is the effect of the intervention on the proportion of women with FGR remaining undelivered by 40 weeks' gestation?

- What is the acceptability of such a package of care to pregnant women and their healthcare providers?

- What other process outcomes are influenced by the intervention, such as healthcare provider/patient interactions?

\section{ENDPOINTS}

\section{Primary outcome}

The primary endpoint is stillbirth (antepartum and intrapartum). We will use the UK definition of stillbirth which is 'a baby delivered without signs of life after $23^{+6}$ weeks. ${ }^{4}$ Where gestation is uncertain we will include all babies with a birth weight of $500 \mathrm{~g}$ or more.

\section{Secondary endpoints}

Other measures of perinatal mortality including:

- stillbirth at 37 weeks' gestation and above;

- stillbirth at 28 weeks' gestation and above (WHO definition of stillbirth);

- stillbirth at 22 weeks' gestation and above (international stillbirth alliance definition);

- stillbirths among normally formed infants of 22 weeks' gestation and above, 24 weeks' gestation and above, 28 weeks' gestation and above and 37 weeks' gestation and above;

- perinatal mortality (defined as stillbirth at 24 weeks' gestation and above and deaths in the first 7 days of life);

- rates of caesarean section;

- rates of induction of labour (for any indication);

- rates of elective delivery (induction of labour and caesarean section prior to the onset of labour) overall;

- rates of induction of labour at 39 weeks' gestation or later;

- mean gestation at induction of labour;

- rates of admission to the neonatal unit (and their reasons);

- rates of admission to the neonatal unit for more than 48 hours;

- rates of admission to the neonatal unit for term babies (those born at 37 weeks 0 days or greater);

- proportion of infants with FGR (less than the fifth centile, customised for gender) remaining undelivered at or after 40 weeks' gestation;

- birth weight centile (according to the Intergrowth birth weight centile calculator at https://intergrowth21.tghn.org);

- rates of spontaneous vaginal delivery.

Other secondary outcomes are the baby parameters:

- gestation at birth

- proportion of babies born preterm $(<37$ weeks' gestation)

- gender of the baby

- birth weight of the baby 
- Apgar score at $5 \mathrm{~min}$

- proportion of babies with 5 min Apgar score $<7$

- proportion of babies with 5 min Apgar score $<4$

- resuscitation required at birth.

We will also collect the following data: maternal age, maternity unit of delivery, birth weight, gestation of delivery, parity, gestation, sex, smoking (current and ever), maternal body mass index (BMI), number of babies (one or more), ethnicity (to allow a customised birth weight centile to be generated), method of delivery, deprivation category (where available) and other neonatal variables including Apgar score and encephalopathy. Adjustment will be made for the following variables: maternal age, maternity unit of delivery, parity, smoking status, maternal BMI, number of babies (one or more) and ethnicity.

\section{STUDY DESIGN}

This is a multicentre, stepped-wedge cluster randomised trial of a package of care consisting of a management plan for identification and delivery of the 'at risk' fetus, together with strategies for increasing pregnant women's awareness of the need to report RFM early. The trial developed from a planned quality improvement project proposed by the Scottish Government to reduce stillbirths. This was planned to emphasise the importance of fetal movement monitoring and was to be rolled out to all NHS maternity units in Scotland. However, prior to this change it was agreed that the roll-out could be performed in such a way as to allow the assessment of the effect of the intervention, the stepped-wedge design would be the natural choice in this circumstance.

The study will take place in participating hospitals in the UK and Ireland (a complete list is available at http://www.crh.ed.ac.uk/affirm/randomised-hospitals/). A nested qualitative study will examine the acceptability of the intervention to patients and healthcare providers and identify process issues (barriers to implementation). Clinical audit (detailed in online supplementary file 2) conducted after the change in practice will be used to determine the effect of interventions on process outcomes (eg, number of women presenting with RFMs, interval between perceiving RFMs and presentation to hospital, number of ultrasound scans, number of admissions for induction of labour). A diagram indicating randomisation of hospital groupings in the stepped-wedge design is shown in figure 1.

The interventions will be introduced over a 32-month period. Data will be collected over a 36-month period. Data in the 'active phase' after introduction of the intervention will be compared with data in the 'control phase'-the period during which usual care processes in study sites are followed from study start to the time of introduction of the intervention. Given that it will take individual units some time (A) to effect change in management in their unit from time of introduction of the intervention and (B) that it will take some time for this change in practice to impact on clinical outcomes, we plan a 'washout' period of 2 months after the introduction of the intervention during which data will not be included in either group for analysis (figure 1). Data will be collected 4 months after the last birth, a further 2 months has been included for data analysis, giving a total study duration of 42 months.

\section{STUDY POPULATION \\ Number of participants}

Participants will be those delivering at all the sites over the study period (36 months). All eligible women will be recruited to the cluster randomised controlled trial. Based on previous delivery numbers, after accounting for a washout period of 2 months (and assuming no withdrawals or losses to follow-up) this is estimated to be a total of around 143140 women per annum. A subset of around 30 participating women and 30 midwives, sonographers and obstetricians will be recruited to the nested qualitative study, which is based in the Scottish sites.

\section{Inclusion criteria}

We will include all women delivering at one of the participating maternity units for the duration of the study. Women who have been seen at any of the maternity units but who deliver at home will not be included. The duration of the study will be 42 months from the start of the trial (1 February 2014). For practical reasons, participants

\begin{tabular}{|c|c|c|c|c|c|c|c|c|c|}
\hline \multirow{2}{*}{$\begin{array}{l}\text { Hospital } \\
\text { groupings }\end{array}$} & \multicolumn{9}{|c|}{ Months since Start of Trial } \\
\hline & $1-4$ & $5-8$ & $9-12$ & $13-16$ & $17-20$ & $21-24$ & $25-28$ & $29-32$ & $33-36$ \\
\hline $1-3$ & & & & & & & & & \\
\hline $4-6$ & & & & & & & & & \\
\hline $7-9$ & & & & & & & & & \\
\hline $10-12$ & & & & & & & & & \\
\hline $13-15$ & & & & & & & & & \\
\hline $16-18$ & & & & & & & & & \\
\hline $19-21$ & & & & & & & & & \\
\hline $22-24$ & & & & & & & & & \\
\hline
\end{tabular}

Figure 1 Stepped-wedge design. The shaded areas (both light and dark) indicate periods in which the interventions are being implemented. The lighter areas indicate the 'transition' period during which data will not be collected for the control or intervention group. The order in which hospital groupings implement the interventions will be determined via randomisation. 
for the nested qualitative study will be recruited from the participating units in Scotland.

\section{Exclusion criteria}

We will exclude women as follows:

- women for whom data on delivery outcomes are still unavailable 4 months after the date of delivery;

- women delivering in the 'washout' period in each unit.

Members of the trial management group and participants who do not speak/understand English will be excluded from participating in the nested qualitative study.

\section{Identifying participants}

Women will be identified from those whose data are included in routine data returns from each unit. Potential participants for the nested qualitative study will be identified from those attending antenatal clinics in participating hospitals, and/or local staff.

\section{Consenting participants}

The main study is a stepped-wedge cluster randomised trial of a package of care which would be introduced in many of the participating units regardless of whether the trial was ongoing or not and the trial uses only routinely collected data on participants. The ethics committee indicated that formal individual patient consent is not necessary for the main trial. Participants in the nested qualitative study will be asked for individual consent.

\section{Screening for eligibility}

As participants are not directly recruited we will not perform any specific screening tests for this aspect of this project. Participants for the nested qualitative study will be: (1) Pregnant women attending hospitals who are participating in the main trial in Scotland. Purposive sampling will ensure that the final sample set includes women who have and who have not experienced RFM, both before and after the introduction of the intervention; (2) Hospital staff (including midwives, ultrasonographers and obstetricians/radiologists) working in participating hospitals in Scotland. There will be no specific screening tests for eligibility for the nested qualitative study, except that women who have experienced a stillbirth in the index pregnancy will not be approached.

\section{Ineligible and non-recruited participants}

Potential participants for the nested qualitative study who are not approached or who decline will have no specific interventions/procedures.

\section{Withdrawal of study participants}

The nature of a cluster randomised study is such that it is not possible for the participant to withdraw from the 'cluster' unless she changes maternity unit part way through her pregnancy. We plan to collect routinely recorded anonymised data; patients have the right to opt out of having their data used-if this happens their data would be excluded from the study database (eg, under the Confidentiality and Security advisory Group Report 2002 and the Data Protection Act (1998) requirements for fair processing of data). Participants in the nested qualitative study who wish to withdraw will be allowed to do so. Their data will be retained and used, unless they additionally indicate that they wish to withdraw their data.

\section{RANDOMISATION}

\section{Randomisation procedures}

This is a cluster-randomised, stepped-wedge design trial wherein maternity units rather than individual patients are randomised. All units will implement the fetal movement monitoring intervention at some point during the trial; the random element is the time point at which this will occur, the so-called 'step' of the stepped-wedge design. Participating maternity units will be blinded to their randomly allocated time point until the time this is required to be revealed to enable the necessary training in the implementation of the intervention to be delivered. Primary and secondary outcomes of the trial will be gathered in a blinded manner via routinely collected data sources.

Maternity units which are in close proximity to each other will be grouped for the purposes of randomisation. This will assist with the feasibility of delivering the training for and implementation of the intervention. Furthermore, this local synchronisation of the intervention implementation will minimise the chances of contamination (introduction of the intervention prematurely) from maternity units which have already implemented the intervention to those not yet randomised.

The order in which the groups of maternity units step in to implement the intervention will be determined by computer-generated random numbers from a uniform distribution. The randomisation list will be held by the Edinburgh Clinical Trials Unit. The identities of the research team staff whose roles in the trial require them to be unblinded to randomisation codes will be recorded in the trial master file (TMF).

\section{Treatment allocation}

Participating sites will be randomised to different schedules for implementing the intervention. All units will be providing conventional treatment at baseline according to local practice-this is the treatment established before the study starts. Sites will be randomised to 'active' treatment in turn as described above. Active treatment will consist of a package of care consisting of a management plan for identification and delivery of the 'at risk' fetus, together with strategies for increasing pregnant women's awareness of the need to report RFM early. The recommended management plan for identification and delivery of the 'at risk' fetus is shown in figure 2. Practice change in the active units will be achieved by: (1) written/email information to all clinicians (doctors, midwives and ultrasonographers) in each unit about the study protocol and amendment of the local 


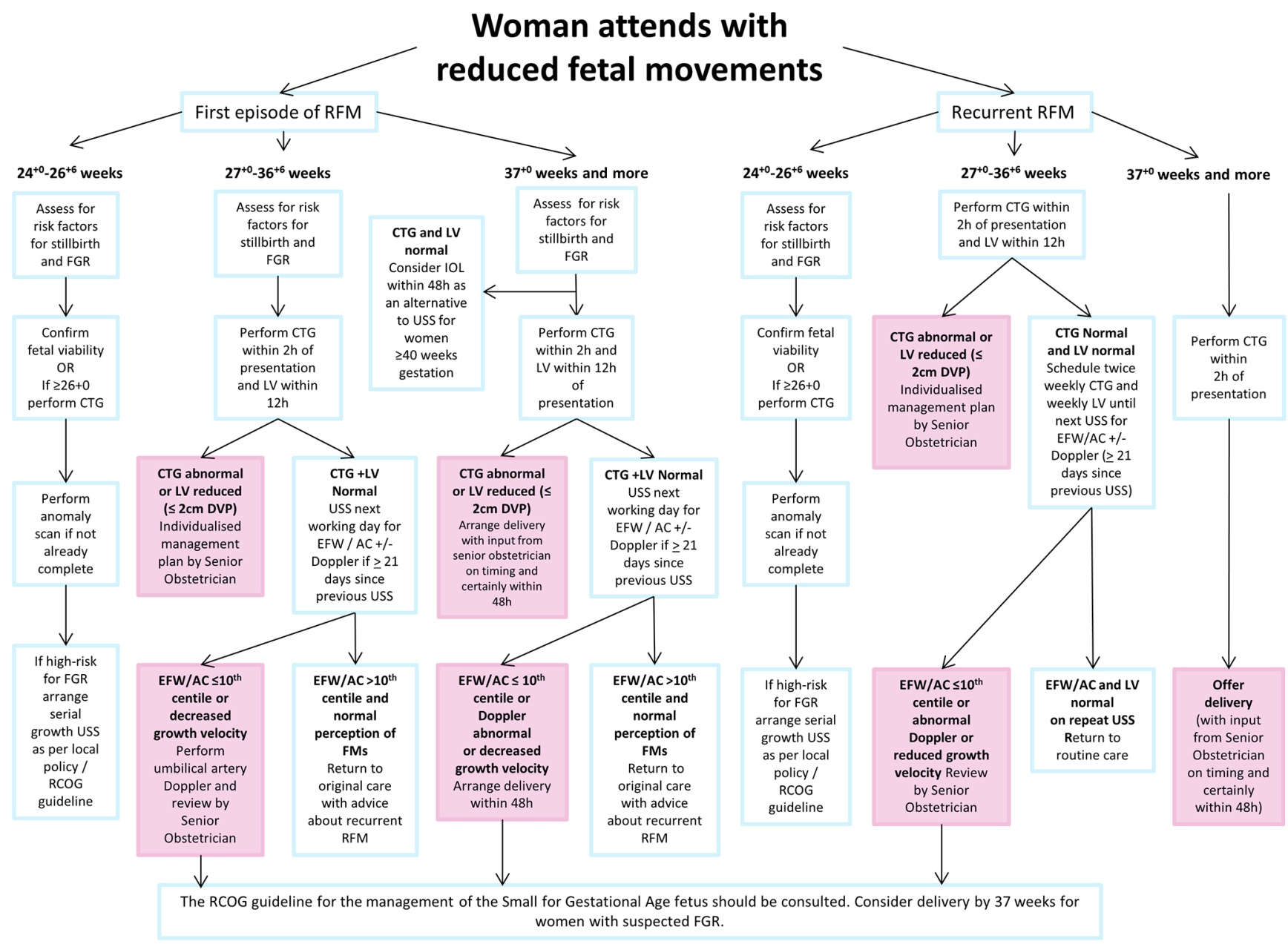

Figure 2 Flow chart for the management of women presenting with RFMs for sites implementing the AFFIRM study. AC, abdominal circumference; CTG, cardiotocography; DVP, deepest vertical pool; EFW, estimated fetal weight; FGR, fetal growth restriction; FM, fetal movement; IOL, induction of labour; LV, liquor volume; RCOG, Royal College of Obstetricians and Gynaecologists; RFM, reduced fetal movement; USS, ultrasound scan.

protocol for RFMs to that of the study protocol; (2) a short web-based training package taking approximately 1 hour to complete for all clinicians in each centre and (3) training/ information sessions to run in each unit and (4) posters in each unit to describe the practice change. Strategies for encouraging clinicians to increase pregnant women's awareness of fetal movement will include all the above and also a fetal movement leaflet for pregnant women (shown in online supplementary file 1). The Norwegian quality improvement study showed inconclusive results regarding the effect of the intervention in non-European women. ${ }^{41}$ To attempt to address this, the AFFIRM information leaflet was available in 12 languages including: Arabic, Bengali, English, Hindi, Hungarian, Latvian, Lithuanian, Mandarin, Polish, Russian and Urdu. Furthermore, by including staff education which highlighted the need to ask women about fetal movements in routine antenatal consultations as many women as possible should have received information about what to do if they perceive RFM.
Once units have begun active treatment it is not anticipated that they will return to conventional treatment. We will conduct an audit of women presenting with RFMs and assess the proportion of staff completing the online training to assess the extent to which sites have followed the intervention plan. Units will be informed about treatment allocation as near as possible to the implementation of the 'active' treatment. For practical purposes, we anticipate that each unit will need around 3 months' notice before the 'active' treatment is introduced, hence units will be informed of the timing of their treatment allocation (step) 3 months before the active treatment is due to start. The treatment allocation will not be administered blind and there are no restrictions on concomitant care or other interventions during the study, hence there is no need for emergency unblinding and there are no stopping rules for the study. 


\section{DATA COLLECTION}

For the main trial, data will be accessed from the information routinely collected during the clinical management of the patient. For consistency, we will normally only include data items which become available within 4 months after the delivery date in question, although we may seek advice from the independently chaired trial steering committee (TSC) about exceptions as they arise. Different data sources will be used for different regions of the study: (1) In Scotland the source data will be SMR2 and the Scottish Birth record, (2) In Ireland the source data will be the National Perinatal Reporting System (NPRS http://www.hpo.ie), (3) In Northern Ireland, the source data will be the Northern Ireland Maternity Statistics database (NIMATS), (4) In England and Wales, the source data will be the Office of National Statistics, or other relevant body. Data will be collected retrospectively on an annual basis from all sources. We will assume that data unavailable 4 months after the woman delivered are likely to be unobtainable (but see note in the Study design section). Thus, data on the first year of the study will be collected at month 16 ; data on the second year will be collected at month 28; and so on.

Data are routinely collected. A formal request for data access will be made at the start of the study. This will require (1) in Scotland-Privacy Advisory Committee approval and a formal approach to NHS Scotland Information Services Division, (2) in Ireland a formal approach to NPRS, (3) NIMATS in Northern Ireland, (4) in England and Wales a formal approach will be made to the relevant bodies.

Data will then be sent to the electronic Data Research and Innovation Service National Safe Haven (NHS National Services Scotland) by secure file transfer protocol (or other similar) for storage and subsequent analysis within a secure project area (dedicated to the AFFIRM study). Further information on the National Safe Haven is available at http://www.isdscotland.org/ Products-and-Services/eDRIS/Becoming-an-eDRISUser/\#NSS-National-Safe-Haven. Briefly, the National Safe Haven is located on a secure server, in which trusted and authorised researchers can analyse individual level data while maintaining the utmost confidentiality. It is anticipated that all study analyses will be done within the Safe Haven, using one of the available statistical packages (eg, R, SPSS Version 23).

Identifiers on Scottish data within the National Safe Haven are concealed from researchers. Data from outwith Scotland will be anonymised before submission to the National Safe Haven. We propose that data submitted to the National Safe Haven will be 'anonymised' by the data provider. However, we propose that the anonymisation link will be retained at the source so that it will be possible to relink data retrospectively. The rationale for retaining the ability of local data guardians to relink data is because it is important to retain the possibility of identifying individual patients retrospectively. Examples include: (1) It is possible that some additional important data may be available at a late stage on individual participants-for example, in the scenario where the woman or baby had a major adverse event and spent a long time in hospital before discharge or death and (2) Although our protocol and outcome analysis does not require identifiable data, we believe this will be a 'once in a lifetime' study, and that subsequent secondary analyses could yield important information for patients and for policymakers. If retrospective identification is not possible, this will limit further analysis. One likely example of future analyses is to determine the effect of the intervention on different causes of stillbirth. This is outwith the scope of the current protocol, but could be done relatively straightforwardly, by linking nationally recorded information on 'cause' of stillbirth to our study database. We anticipate that such additional analyses would require additional ethics approval, but without a process by which to relink data, it will not be possible to perform such subsequent analyses.

All investigators and study site staff involved with this study will comply with the requirements of the Data Protection Act 1998 (or equivalent for those outwith the UK) with regard to the collection, storage, processing and disclosure of personal information and will uphold the Act's core principles. Published results will not contain any personal data that could allow identification of an individual participant.

In addition to the data recorded above, all sites will be asked to provide a copy of their guidelines around (1) maternal awareness of RFM and (2) management of women presenting with RFM. Copies of guidelines will be sought by the study office (A) at the start of the study, (B) immediately before initiation of the intervention in each specific unit and (C) 6 months after initiation of the intervention in each specific unit.

For the nested qualitative study, we will perform interviews of healthcare workers and a small nested cohort of pregnant women about their experiences of fetal movement and of this intervention. We shall ensure a diversity of age and include nulliparous and multiparous women $(\mathrm{n}=30$ in total). Ten interviews will be conducted with each of the following groups of healthcare providers: obstetricians, midwives and sonographers/radiologists. The interviews will take a semistructured format (sensitising and piloting interviews will be conducted prior to the commencement of the trial and in the first month of the nested qualitative study). This format will ensure the same categories of data will be obtained from each participant but also allow individual responses to be fully explored.

\section{STATISTICS AND DATA ANALYSIS}

\section{Sample size calculation}

The sample size is the number of women delivering in hospitals participating in the study. This was initially planned to include sites in Scotland, totalling around 
58000 deliveries per year with 16 consultant-led maternity units, 20 smaller units each delivering less than 350 babies per year, and seven units delivering less than five births per year. The units involved in Perinatal Ireland (an all-Ireland research consortium across seven academic sites in Ireland currently funded by the Health Research Board, Ireland) have 50000 births per year with seven large sites. Combining one or two of the smaller units and one larger unit into a single 'hospital group' for each local area could provide 24 hospital 'groups'the details of hospital groupings will be reviewed and finalised immediately prior to randomisation. In total, 36 sites expressed interest in participating in the study, although two were unable to participate in the study and withdrew before randomisation. In total, 34 units were randomised, these were situated throughout the UK and Ireland (10 in England, 4 in Ireland, 15 in Scotland and 5 in Wales) with 143140 births per annum.

We calculated statistical power using the methodology for stepped-wedge designs proposed in Hussey and Hughes (2007). ${ }^{42}$ First, we analysed stillbirth event data from the Scottish Perinatal and Infant Mortality and Morbidity Report covering years 2005-2010 ${ }^{17}$ to determine estimates of between-unit and within-unit variability in stillbirth rate. Analysis was by generalised linear mixed model for binary outcomes. The power calculation, as per equations (\#7) and (\#8) in reference 42 assumed: significance level $5 \%$; analysis by generalised linear mixed model; deliveries equally distributed across hospital groupings; baseline stillbirth rate $0.438 \%{ }^{17}$; cluster coefficient of variation 0.333 .

Finally, the statistical power depends on the number of groups in which the intervention is implemented at each stage of the stepped-wedge design and the duration of recruitment at each 'step.' Our study design proposes sequential introduction of the intervention into three hospital groups at a time in eight steps at 4 -month intervals. This would give $92.4 \%$ power to detect a $30 \%$ risk reduction under the intervention and $80.7 \%$ power to detect a $25 \%$ reduction. A $30 \%$ risk reduction was seen in the Norwegian study; the anticipated effect sizes of $25 \%$ and $30 \%$ relative reduction take into account that the intervention will not have the power to reduce all stillbirths, since $20 \%$ of stillbirths in Ireland ${ }^{43}$ and $15 \%$ in Scotland ${ }^{17}$ are associated with congenital anomaly.

The power actually achieved in the study will be slightly lower, as deliveries during the 2-month 'transition' period following implementation of the intervention in a site will not be included in the analysis. The effect of this was explored using the Stata function steppedwedge, ${ }^{44}$ which showed the statistical power would become $88.2 \%$ (30\% risk reduction) and $74.6 \%$ (25\% risk reduction). It is anticipated that unavailability of data and women asking to withdraw their data will be less than $1 \%$.

\section{Proposed analyses}

For the binary primary and secondary outcomes, data will be analysed by generalised linear mixed model with a random effect for hospital and fixed effects for the intervention implementation and study time period. A site by intervention interaction random effect will be included in the model and retained if it explains an important proportion of the variability in outcomes. The primary analysis of data will be on an intention to treat basis (the design of the trial means it is not possible to determine individual patient/caregiver compliance with the intervention). An 'on treatment' variable will be calculated for which women will be grouped as active or control according to when the intervention was actually implemented in their site, instead of when the site was randomised to implement the intervention. The primary outcome will be reanalysed in two sensitivity analyses. First, we will perform the analysis according to the actual timing of the implementation of the intervention rather than the randomised timing of the intervention using the 'on treatment' classification. Second, we will perform the analysis in the subgroup of sites who were deemed to have implemented the intervention effectively according to the perception of the Principal Investigator at each site. The accuracy of this perception will be confirmed with the findings of a site audit (details in online supplementary file 2). There will be no attempt to correlate the impact of the intervention according to the results of the site audit.

There are no planned imputations for missing data. However, if the missing data rate for smoking status during pregnancy is relatively high an imputation technique will be devised. The imputation method will be informed using smoking history at booking and age at delivery. ${ }^{45}$ A prespecified subgroup analysis will be performed for babies with and without congenital anomalies, and will be implemented by testing for an intervention by congenital anomaly interaction added to the generalised linear mixed model described above. No formal interim analyses for efficacy or safety will be performed. A full statistical analysis plan will be finalised prior to locking of the study database.

\section{Qualitative data}

For the nested qualitative study, the data will be audio-recorded and transcribed. The data will be coded thematically and an analytical framework developed to make sense of patient experience of fetal movement and the intervention and also healthcare providers' perspectives and experiences. NVivo will be used to support the analysis.

\section{Process outcomes}

The process outcomes being assessed by the rates of induction of labour, number of women presenting with RFMs, interval between perceiving fetal movements and presenting to hospital will be analysed using the same methods as for the main trial, with the exception of the 
continuous outcome (interval between perceiving fetal movements and presenting to hospital) which will be analysed using a normal linear mixed model.

\section{ADVERSE EVENTS}

This is not a Clinical Trial of an Investigational Medicinal Product (CTIMP) so adverse events will not be formally reported. Stillbirth and other measures of fetal and maternal morbidity are outcomes of the study. The purpose of the intervention is to reduce such adverse events. Therefore, due to the low risks for this trial, a separate Data Monitoring Committee (DMC) is not required and the TSC will cover any responsibilities normally allocated to a DMC. If considered necessary, the TSC may review unblinded data for the study, including morbidity and mortality indices. No other adverse event reporting will be undertaken.

\section{TRIAL MANAGEMENT AND OVERSIGHT ARRANGEMENTS}

The trial will be coordinated by a Project Management Group, consisting of the grant holders and the Trial Manager. The Chief Investigator (JEN) will lead the project management group. The Trial Manager will oversee the study and will be accountable to the Chief Investigator. A TSC will be established to oversee the conduct and progress of the trial. The terms of reference and a draft template for reporting will be ratified in one of the early meetings of the TSC.

Investigators and institutions involved in the study will permit trial-related monitoring and audits on behalf of the cosponsors (Academic and Clinical Central Office for Research \& Development-joint office for University of Edinburgh and NHS Lothian, Sponsor contact: ray.french@ed.ac.uk), research ethics committee (REC) review and regulatory inspection(s). In the event of an audit or monitoring, the Investigator agrees to allow the representatives of the sponsor direct access to all study records and source documentation. In the event of regulatory inspection, the Investigator agrees to allow inspectors direct access to all study records and source documentation.

\section{Study monitoring and audit}

The sponsor determined that as no individual participants were recruited to the intervention, and it was not a CTIMP no formal monitoring and audit was required.

\section{Good clinical practice and ethical conduct}

The study will be conducted in accordance with the principles of the research governance framework operational and good clinical practice in the relevant country. A favourable ethical opinion has been obtained from the Scotland A REC (Reference 13/SS/0001) and local research and development approval has been obtained prior to commencement of the study.
Local study investigator(s) will be appointed to each site (or for small units, groups of sites). She/He will be responsible for the overall conduct of the study at the site and compliance with the protocol and any protocol amendments.

\section{STUDY CONDUCT RESPONSIBILITIES \\ Protocol amendments}

Any changes in research activity, except those necessary to remove an apparent, immediate hazard to the participant in the case of an urgent safety measure, will be reviewed and approved by the Chief Investigator and Sponsor. Amendments to the protocol will be submitted in writing to the appropriate REC and local Research and Development (R\&D) department for approval prior to participants being enrolled into an amended protocol.

\section{Protocol violations and deviations}

Investigators will not implement any deviation from the protocol without agreement from the Chief Investigator and appropriate REC and R\&D department approval except where necessary to eliminate an immediate hazard to trial participants. In the event that an Investigator needs to deviate from the protocol, the nature of and reasons for the deviation will be recorded. If this necessitates a subsequent protocol amendment, this will be submitted to the REC, and local R\&D department for review and approval if appropriate.

\section{Serious breach requirements}

A serious breach is one which is likely to effect to a significant degree (A) the safety or physical or mental integrity of the participants of the trial; or (B) the scientific value of the trial. If a potential serious breach is identified by the Chief investigator, Principal Investigator or delegates, the cosponsors (accord.seriousbreach@ed.ac.uk) will be notified within 24 hours. It will be the responsibility of the cosponsors to assess the impact of the breach on the scientific value of the trial, to determine whether the incident constitutes a serious breach and, if so, report it to the REC.

All violations will be assessed by the sponsor(s) to ascertain if they meet the criteria for a serious breach. If the sponsor(s) deem the incident to be a violation that does not constitute a serious breach from the protocol when identified, corrective and preventative actions will be taken where appropriate and they will be recorded in file notes, held within the TMF and Investigator Site File.

\section{Study record retention}

All study documentation will be kept for a minimum of 5 years from the protocol defined end of study point. When the minimum retention period has elapsed, study documentation will not be destroyed without permission from the sponsor. 


\section{End of study}

The end of study date was finalised in the protocol after the study commenced; the agreed end of study date is 31 December 2016. The Investigators and/or the TSC and/or the cosponsor(s) have the right at any time to terminate the study for clinical or administrative reasons.

The end of the study will be reported to the REC within 90 days, or 15 days if the study is terminated prematurely. The Investigators will inform participants of the premature study closure and ensure that the appropriate follow-up is arranged for all participants involved. A summary report of the study will be provided to the REC and Regulatory Authority within 1 year of the end of the study.

\section{REPORTING, PUBLICATIONS AND NOTIFICATION OF RESULTS}

Ownership of the data arising from this study resides with the study team. On completion of the study, the study data will be analysed and tabulated, and a clinical study report will be prepared in accordance with good clinical practice guidelines. The clinical study report will be used as the basis for publication and presentation at scientific meetings. Investigators have the right to publish orally or in writing the results of the study. Summaries of results will also be made available to Investigators for dissemination within their clinics (where appropriate and according to their discretion).

\section{DISCUSSION}

The data provided by this study will inform the information given to women about RFMs and their management when they present to maternity services; which has been recurrently identified by Confidential Enquiries into antepartum stillbirths as suboptimal. ${ }^{27}{ }^{28}$ Data from the AFFIRM study will be able to be compared with results from two other active studies which aim to improve mothers' awareness and reporting of RFMs. My Babies Movement (ACTRN 12614000291684) is a stepped-wedge cluster trial of a mobile phone application to help women get to know their babies' movements, to be mindful of movements every day and not to wait to report concerns to their maternity care provider. The Mindfetalness study (NCT02865759) is a cluster trial of 39000 women randomised to routine antenatal care or the Mindfetalness brochure and website. ${ }^{46}$ Women participating in the Mindfetalness process will spend 15 min each day getting to know their babies' movements and will specifically be encouraged to contact their health provider if they perceive RFMs. This primary outcome of this study is an Apgar score $<7$ at $5 \mathrm{~min}$; stillbirth and perinatal deaths will be recorded as tertiary endpoints of this study. ${ }^{46}$ These large studies will provide much needed robust evidence to determine whether increased maternal awareness of RFMs combined with a standardised management protocol to identify acute or chronic fetal compromise can reduce stillbirth. ${ }^{33}$

\section{Author affiliations}

${ }^{1}$ Maternal and Fetal Health Research Centre, School of Medical Sciences, Faculty of Biology, Medicine and Health, University of Manchester, Manchester, UK

${ }^{2}$ St. Mary's Hospital, Central Manchester University Hospitals NHS Foundation Trust, Manchester Academic Health Science Centre, Manchester, UK

${ }^{3}$ Edinburgh Clinical Trials Unit, Edinburgh, UK

${ }^{4}$ Centre for Population Health Sciences, Usher Institute of Population Health Sciences and Informatics, University of Edinburgh, Teviot Place, Edinburgh, UK

${ }^{5}$ Tommy's Centre for Maternal and Fetal Health, MRC Centre for Reproductive Health, Queen's Medical Research Institute, Edinburgh, UK

${ }^{6}$ School of Women's and Infants' Health, University of Western Australia, Crawley, Western Australia, Australia

${ }^{7}$ The Scottish Government St Andrew's House, Edinburgh, UK

${ }^{8}$ Department of International Public Health, Norwegian Institute of Public Health, Nydalen, Oslo, Norway

${ }^{9}$ Rotunda Hospital, Parnell Square, Dublin, Ireland

${ }^{10}$ Centre for Fetal Medicine, Royal Maternity Hospital, Belfast, UK

${ }^{11}$ UCD Obstetrics \& Gynaecology, School of Medicine, University College Dublin, National Maternity Hospital, Dublin, Ireland

${ }^{12}$ Department of Neonatology, Royal Infirmary of Edinburgh, NHS Lothian, Edinburgh, UK

${ }^{13} \mathrm{NHS}$ Education for Scotland, Edinburgh, UK

${ }^{14}$ Sands, Victoria Charity Centre, London, UK

Acknowledgements The authors would like to acknowledge the support of Perinatal Ireland and Dr Mary Higgins (University College Dublin, National Maternity Hospital, Dublin).

Contributors AEPH, CJW, SJES, CJC, SCB, MRD, SW and JEN were involved in developing the trial design. AEPH, CJW, SJES, AR and JEN were involved in drafting and revision of the article. CJW and AR were involved in drafting the statistical aspects of the protocol. JS provided feedback on behalf of a stakeholder organisation. AEPH, CJW, SJES, CJC, SCB, JFF, MG, AH, FMM, EM, AR, MRD, JS, SW and JEN were involved in preparing the overall study design. AEPH, JEN and MRD prepared education videos for online training. AEPH, SJES, SJS, MG, AH, FMM and JEN facilitated recruitment of sites. AEPH, CJW, SJES, CJC, SCB, JFF, MG, AH, FMM, EM, AR, MRD, JS, SW and JEN will be involved in the collection, management, analysis and interpretation of data, and final writing of the trial report.

Funding The AFFIRM study is investigator initiated and funded by Chief Scientist Office, Scottish Government (CZH/4/882), Tommy's and Sands, the Stillbirth and Neonatal Death Charity. CJW was supported in this work by NHS Lothian via the Edinburgh Clinical Trials Unit. AEPH is supported by a Clinician Scientist 801 fellowship from the National Institute for Health Research (NIHR; CS-2013-009). This protocol presents independent research funded by the National Institute for Health Research (NIHR). The views expressed are those of the author(s) and not necessarily those of the NHS, the NIHR or the Department of Health.

Competing interests None declared.

Ethics approval Scotland A REC: 13/SS/0001.

Provenance and peer review Not commissioned; externally peer reviewed.

Open Access This is an Open Access article distributed in accordance with the Creative Commons Attribution Non Commercial (CC BY-NC 4.0) license, which permits others to distribute, remix, adapt, build upon this work non-commercially, and license their derivative works on different terms, provided the original work is properly cited and the use is non-commercial. See: http://creativecommons.org/ licenses/by-nc/4.0/

(C) Article author(s) (or their employer(s) unless otherwise stated in the text of the article) 2017. All rights reserved. No commercial use is permitted unless otherwise expressly granted.

\section{REFERENCES}

1. Still-Birth. Definition Act Great Britain. Curr Law Statut Annot GB 1992:1.

2. Flenady V, Wojcieszek AM, Middleton P, et al. Stillbirths: recall to action in high-income countries. Lancet 2016;387:691-702.

3. Flenady V, Koopmans L, Middleton P, et al. Major risk factors for stillbirth in high-income countries: a systematic review and metaanalysis. Lancet 2011;377:1331-40. 
4. Manktelow BM, Smith LK, Seaton SE, et al. Perinatal Mortality Surveillance Report - UK Perinatal Deaths for births from January to December 2014. Leicester: The Infant Mortality and Morbidity Group, Department of Health Sciences, University of Leicester., 2016.

5. Lawn JE, Blencowe H, Pattinson R, et al. Lancet's Stillbirths Series steering committee. Stillbirths: Where? When? Why? How to make the data count? Lancet 2011;377:1448-63.

6. Flenady V, Middleton P, Smith GC, et al. Stillbirths: the way forward in high-income countries. Lancet 2011;377:1703-17.

7. Heazell $\mathrm{AE}$, Whitworth MK, Whitcombe J, et al. Research priorities for stillbirth: process overview and results from UK Stillbirth Priority Setting Partnership. Ultrasound Obstet Gynecol 2015;46:641-7.

8. Heazell AE, Frøen JF. Methods of fetal movement counting and the detection of fetal compromise. J Obstet Gynaecol 2008;28:147-54.

9. Stacey T, Thompson JM, Mitchell EA, et al. The Auckland Stillbirth study, a case-control study exploring modifiable risk factors for third trimester stillbirth: methods and rationale. Aust N Z J Obstet Gynaecol 2011;51:3-8.

10. Warland J, O'Brien LM, Heazell AE, et al. STARS Consortium. An international internet survey of the experiences of 1,714 mothers with a late stillbirth: the STARS cohort study. BMC Pregnancy Childbirth 2015;15:172.

11. Warrander LK, Heazell AE. Identifying placental dysfunction in women with reduced fetal movements can be used to predict patients at increased risk of pregnancy complications. Med Hypotheses 2011;76:17-20.

12. Vintzileos AM, Fleming AD, Scorza WE, et al. Relationship between fetal biophysical activities and umbilical cord blood gas values. $A m J$ Obstet Gynecol 1991;165:707-13.

13. Warrander LK, Batra G, Bernatavicius G, et al. Maternal perception of reduced fetal movements is associated with altered placental structure and function. PLoS One 2012;7:e34851.

14. Winje BA, Roald B, Kristensen NP, et al. Placental pathology in pregnancies with maternally perceived decreased fetal movement-a population-based nested case-cohort study. PLoS One 2012;7:e39259.

15. Holm Tveit JV, Saastad E, Stray-Pedersen B, et al. Maternal characteristics and pregnancy outcomes in women presenting with decreased fetal movements in late pregnancy. Acta Obstet Gynecol Scand 2009;88:1345-51.

16. Ptacek I, Sebire NJ, Man JA, et al. Systematic review of placental pathology reported in association with stillbirth. Placenta 2014;35:552-62.

17. Healthcare Improvement Scotland. Scottish Perinatal and Infant Mortality and Morbidity Report 2010. Edinburgh: Healthcare Improvement Scotland. 2012.

18. Gardosi J, Kady SM, McGeown P, et al. Classification of stillbirth by relevant condition at death (ReCoDe): population based cohort study. BMJ 2005;331:1113-7.

19. Saastad E, Winje BA, Stray Pedersen B, et al. Fetal movement counting improved identification of fetal growth restriction and perinatal outcomes-a multi-centre, randomized, controlled trial. PLOS One 2011;6:e28482.

20. Mangesi L, Hofmeyr GJ, Smith V, et al. Fetal movement counting for assessment of fetal wellbeing. Cochrane Database Syst Rev 2015;10:CD004909.

21. Grant A, Elbourne D, Valentin L, et al. Routine formal fetal movement counting and risk of antepartum late death in normally formed singletons. Lancet 1989;2:345-9.

22. National Institute for Health and Clinical Excellence. Clinical Guideline 62 - Antenatal care: routine care for the health pregnant woman. London: National Institute for Health and Clinical Excellence, 2008.

23. Sergent F, Lefèvre A, Verspyck E, et al. [Decreased fetal movements in the third trimester: what to do?]. Gynecol Obstet Fertil 2005;33:861-9.

24. Frøen JF, Heazell AE, Tveit JV, et al. Fetal movement assessment. Semin Perinatol 2008;32:243-6.

25. Saastad E, Vangen S, Frøen JF. Suboptimal care in stillbirths - a retrospective audit study. Acta Obstet Gynecol Scand 2007;86:444-50.
26. Frøen JF, Arnestad M, Frey K, et al. Risk factors for sudden intrauterine unexplained death: epidemiologic characteristics of singleton cases in Oslo, Norway, 1986-1995. Am J Obstet Gynecol 2001;184:694-702.

27. Confidential Enquiry into Stillbirths and Deaths in Infancy. 8th Annual Report, 1 January-31 December 1999. London: Maternal and Child Health Research Consortium, 2001.

28. Draper ES, Kurinczuk JJ, Kenyon S. MBRRACE-UK. obo. MBRRACE-UK Perinatal Confidential Enquiry: Term, singleton, normally formed, antepartum stillbirth. Leicester: The Infant Mortality and Morbidty Studies, Department of Health Sciences, University of Leicester, 2015.

29. Linde A, Pettersson K, Rådestad I. Women's Experiences of Fetal Movements before the Confirmation of Fetal Death--Contractions Misinterpreted as Fetal Movement. Birth 2015;42:189-94.

30. Preston S, Mahomed K, Chadha Y, et al. Clinical practice guideline for the management of women who report decreased fetal movements. Brisbane: Australia and New Zealand Stillbirth Alliance, 2010.

31. Royal College Of Obstetricians and Gynaecologists. Management of Reduced Fetal Movements. London: RCOG, 2011.

32. Tveit JV, Saastad E, Stray-Pedersen B, et al. Reduction of late stillbirth with the introduction of fetal movement information and guidelines - a clinical quality improvement. BMC Pregnancy Childbirth 2009;9:32.

33. Hofmeyr GJ, Novikova N. Management of reported decreased fetal movements for improving pregnancy outcomes. Cochrane Database Syst Rev 2012;4:CD009148.

34. Frøen JF, Tveit JV, Saastad E, et al. Management of decreased fetal movements. Semin Perinatol 2008;32:307-11.

35. Dutton PJ, Warrander LK, Roberts SA, et al. Predictors of poor perinatal outcome following maternal perception of reduced fetal movements--a prospective cohort study. PLoS One 2012;7:e39784.

36. Royal College Of Obstetricians and Gynaecologists. The Investigation And Management Of The Small-For-Gestational-Age Fetus. London: RCOG, 2013.

37. Heazell AE, Green M, Wright C, et al. Midwives' and obstetricians' knowledge and management of women presenting with decreased fetal movements. Acta Obstet Gynecol Scand 2008;87:331-9.

38. Jokhan $\mathrm{S}$, Whitworth MK, Jones $\mathrm{F}$, et al. Evaluation of the quality of guidelines for the management of reduced fetal movements in UK maternity units. BMC Pregnancy Childbirth 2015;15:54.

39. Tveit JVH, Saastad E, Stray-Pedersen B, et al. Erratum to: reduction of late stillbirth with the introduction of fetal movement information and guidelines - a clinical quality improvement. BMC Pregnancy Childbirth 2010;10:49.

40. Winje BA, Wojcieszek AM, Gonzalez-Angulo LY, et al. Interventions to enhance maternal awareness of decreased fetal movement: a systematic review. BJOG 2016;123:886-98.

41. Saastad E, Tveit JV, Flenady V, et al. Implementation of uniform information on fetal movement in a Norwegian population reduced delayed reporting of decreased fetal movement and stillbirths in primiparous women - a clinical quality improvement. BMC Res Notes 2010;3:2.

42. Hussey MA, Hughes JP. Design and analysis of stepped wedge cluster randomized trials. Contemp Clin Trials 2007;28:182-91.

43. ESRI Health Research and Information Division. Perinatal Statistics Report 2009. 2011.

44. Hemming K, Girling A. A menu-driven facility for power and detectable-difference calculations in stepped-wedge clusterrandomized trials. The Stata Journal 2014;14:363-80.

45. Tominey E. Maternal smoking during pregnancy and early child outcomes. Discussion Paper no. 828. London: Centre for Economic Performance, London School of Economics, 2007.

46. Rådestad I, Akselsson A, Georgsson S, et al. Rationale, study protocol and the cluster randomization process in a controlled trial including 40,000 women investigating the effects of mindfetalness. Sex Reprod Healthc 2016;10:56-61. 